\title{
The effects of abdominal drawing-in maneuver during stair climbing on muscle activities of the trunk and legs
}

\author{
Su-Kyoung Lee* \\ Department of Physical Therapy, College of Nursing, Healthcare Sciences \& Human Ecology, Dong-Eui University, Busan, Korea
}

The purpose of this study was to investigate effects of abdominal drawing-in maneuver (ADIM) on the muscular activity of the trunk and the lower limbs during stair ascent. The subjects were healthy 15 men and 8 women who were in their 20s. After maintaining ADIM by using a pressure biofeedback unit for $15 \mathrm{~min}$, the subjects performed stair ascent and their muscular activity of the right trunk and the legs was measured to determine difference between when the subjects maintained ADIM and did not. The activities of the sternocleidomastoid, the splenius capitis, the rectus abdominis (ABD), the external abdominal oblique (EO), the transverse abdominis (TRA), the elector spinae (ES), the vastus medialis (VM), and the vastus lateralis (VL) were measured using surface electromyogram (TM DTS). The collected data were sta-

\section{INTRODUCTION}

The abdominal core muscles, involved in all the forces and movements of the body, control postures and maintain the musculoskeletal structure properly (Akuthota and Nadler, 2004; Marshall and Murphy, 2005; Richardson et al., 2004). The muscles affect the overall postural alignment and block stress put on the waist. As tonic muscles that minimize the shear force applied to the lumbar vertebrae, they play a major role in maintaining the spinal curvature and stability in the anterior, posterior, and lateral vertebrae. The global muscles such as the rectus abdominal and the external oblique muscles generate a wide range of movements and torques of the trunk and the pelvis, contributing to overall stabilization of the trunk; the transverse abdominal and the internal oblique abdominal muscles are involved in stability and fine movements of the spine between the spinal segments (Bergmark, 1989). tistically processed by the SPSS ver. 18.0 and analyzed with a paired $t$-test. The muscular activities of the TRA, VM, and VL significantly increased $(P<0.05)$, while the activity of the ES significantly decreased $(P<0.05)$ during stair ascent when the ADIM was maintained than when it was not. In conclusion, the stair ascent with ADIM maintained activated the abdominal muscles to control trunk balance and perform lumbar stability, simultaneously reducing excessive lumbar lordosis or pelvic anterior tilt which will help to improve the function of the musculoskeletal system.

Keywords: ADIM, Biofeedback, Stairs, Muscle activity
${ }^{*}$ Corresponding author: Su-Kyoung Lee (iD https://orcid.org/0000-0002-4916-2188 Department of Physical Therapy, College of Nursing, Healthcare Sciences \& Human Ecology, Dong-Eui University, 176 Eomgwang-ro, Busanjin-gu, Busan 47430, Korea E-mail: ptlsk@deu.ac.kr

Received: February 1, 2019/ Accepted: March 23, 2019
Thus, the abdominal muscles of these stabilizing muscles act in all movements of the human body by contracting before other muscles to properly balance the imbalance and maintain the balance of the body (Hodges and Gandevia, 2000), increasing the stability of the spine and the pelvis during functional postures and movements, enhancing muscular strength, and recovering balance and control of the muscles and movements (Richardson et al., 2002). Performing stabilization exercise controls contraction of the trunk muscles to restrain degeneration caused by segmental instability, thereby reducing risks of microdamage (Saal, 1990), enhancing muscular strength, and restoring control and balance of muscles and movements (Standaert et al., 2008).

Abdominal drawing-in maneuver (ADIM) as a stabilization exercise functionally stimulates the transverse abdominal, the internal oblique abdominal, and the multifidus muscles (Richardson et al., 2004), selectively contract the transversus abdominis to provide trunk stability (Hodges and Richardson, 1996; Hodges et 
al., 2005). The ADIM is also used to reduce symptoms of patients with lumbar pain (Macedo et al., 2009), serving as a significant key in trunk stabilization and postural control with its abdominal muscular control (Bjerkefors et al., 2010). Walking is a crucial, basic motion in daily life, an activity in which motor skills and trunk movement are performed simultaneously. During walking, a human body in an upright position uses several joints, nerves, and muscles to move the center of the trunk, to keep its balance, and to control its posture (Hubble et al., 2014). Walking is also a highly coordinated alternating movement in which the body moves step by step with the necessary speed maintained toward a certain direction. The legs during walking basically take charge of weight bearing and keep stability and balance while providing basic motions for movement and propelling the body forward (Galley and Foster, 1987). Stair ascent, as well as flatland walking, is a functionally common action in daily life and can be performed easily and with low cost, and yet it possesses several advantages including improved health of the cardiovascular system (Meyer et al., 2009), increased bone density, and prevention of falling by improved balance (Borschmann et al., 2012). Stair ascent, in particular, exhibits biomechanical characteristics in which the extensors of the lower limbs accelerate the body perpendicularly and forward, a proper action to enhance the muscular strength of the trunk and the lower limbs (Riener et al., 2002; Yoon et al., 2009).

Despite on-going research on stair ascent, however, few studies have associated stair ascent with the abdominal core muscles. In this context, we compared the muscular activity during stair ascent between when the subjects maintained ADIM and did not.

\section{MATERIALS AND METHODS}

\section{Participants}

The subjects of this study were healthy 15 male and 8 female students in Dong-Eui University located in Busan, Korea. After explaining the contents and purposes of this study, we chose those who agreed to participation as the subjects. All the subjects had no musculoskeletal diseases for the last three months, were able to walk normally, and had no muscular weakness in their abdomen and legs. We fully explained the purposes and methods of the experiment in advance, and the subjects voluntarily submitted a consent for participation, the participation that was performed in random order. This study was approved by the research ethics committee (DIRB-201803-HR-E-15).

\section{Pressure biofeedlback unit}

The subjects performed the ADIM with a pressure biofeedback unit. They lied down on a stable supporting surface with their knee joints bent at 90 degrees, the units placed under the fifth lumbar vertebra. They were asked to increase and maintained the pressure from $40 \mathrm{mmHg}$ by $10 \mathrm{mmHg}$, which was shown on the gauge connected to the units. During the ADIM, the subjects pulled their navel upward and rearward (toward the lumbar), allowing their abdomen to be slightly concave, like in expiration. They maintained the maneuver for ten seconds, breathing normally. When they repeated the activity ten times and took a 3-min rest, it was counted as a set. Three sets were performed for $15 \mathrm{~min}$.

\section{Surface electromyogram}

Surface electromyogram (EMG), Noraxon telemyo 2400 system (TM DTS, Noraxon, Scottsdale, AZ, USA), was used to measure effects of ADIM on muscular activity during stair ascent. When the subjects took a posture that could give maximum resistance to each muscle, we attached positive electrodes to the muscle belly, the area that was most activated in muscular contraction, and negative electrodes to an area $2 \mathrm{~cm}$ from the positive electrodes, parallel to the length of the muscle. The electrodes were attached to the sternocleidomastoid (SCM), the splenius capitis (SC), the rectus abdominis $(\mathrm{ABD})$, the external abdominal oblique (EO), the transverse abdominis (TRA), the elector spinae (ES), the vastus medialis (VM), and the vastus lateralis (VL). The sampling rate of the EMG signals was set at 3,000 Hz. The measured EMG data were removed of noises by using the frequency band of 20$500 \mathrm{~Hz}$ to find root mean square values. Other signals were processed by rectification and smoothing. In addition, the EMG signals were normalized as the $\%$ reference voluntary contraction that was used as reference values, from normalization of the EMG signals at a certain motion.

\section{Measurement methods}

Before the experiment, the subjects performed the ADIM for $15 \mathrm{~min}$ and then practiced stair ascent once during maintaining or not maintaining the maneuver. In the experiment, we set the order of maintaining and not maintaining the ADIM through a single-blind study and a randomized controlled trial, and the subjects performed stair ascent according to the order for measurement of their muscular activity. In order to prevent substitution, we applied a 3-min rest after three repetitive measurements for each order. 
Table 1. Characteristics of study participants $(n=23)$

\begin{tabular}{lc}
\hline Characteristic & Value \\
\hline Gender, male:female & $15: 8$ \\
Age $(\mathrm{yr})$ & $22.43 \pm 2.01$ \\
Height $(\mathrm{cm})$ & $169.46 \pm 7.63$ \\
Weight $(\mathrm{kg})$ & $68.19 \pm 11.45$ \\
\hline
\end{tabular}

Values are presented as number or mean \pm standard error.

\section{Data analysis}

The IBM SPSS ver. 18.0 (IBM Co., Armonk, NY, USA) was used to analyze the collected data. A paired $t$-test was used to determine muscular activities of the trunk and lower limb muscles during stair ascent based on ADIM. The statistical significance level $(\alpha)$ was set at 0.05 .

\section{RESULTS}

\section{General characteristics of the subjects}

The subjects were 15 men and 8 women. The age was $22.43 \pm$ 2.01 years, the height was $169.46 \pm 7.63 \mathrm{~cm}$, and the weight was $68.19 \pm 11.45 \mathrm{~kg}$. Their general characteristics are as seen in Table 1 .

\section{Comparison of muscular activity \\ Muscular activity of the transverse abdominal based on intervention}

The muscular activity of the transverse abdominal increased during stair ascent significantly more when the ADIM was maintained than when it was not $(P<0.05)$ (Table 2).

\section{Muscular activity of the elector spinae based on intervention}

The muscular activity of the elector spinae decreased during stair ascent significantly more when the ADIM was maintained than when it was not $(P<0.05)$ (Table 2$)$.

\section{Muscular activity of the vastus medialis based on intervention}

The muscular activity of the vastus medialis increased during stair ascent significantly more when the ADIM was maintained than when it was not $(P<0.05)$ (Table 2).

\section{Muscular activity of the vastus lateralis based on intervention}

The muscular activity of the vastus lateralis increased during stair ascent significantly more when the ADIM was maintained than when it was not $(P<0.05)$ (Table 2).
Table 2. Comparison of muscle activity according to presence or absence of abdominal drawing-in maneuver during stair climbing

\begin{tabular}{lcccc}
\hline Type of muscle & Pre & Post & $t$ & $P$-value \\
\hline SCM & $266.47 \pm 34.97$ & $372.40 \pm 92.22$ & -1.28 & 0.21 \\
SC & $259.65 \pm 33.88$ & $269.81 \pm 37.84$ & -0.24 & 0.68 \\
ABD & $404.96 \pm 65.79$ & $478.22 \pm 79.31$ & -1.27 & 0.22 \\
EO & $492.50 \pm 108.67$ & $657.32 \pm 110.99$ & -1.98 & 0.60 \\
TRA & $427.48 \pm 72.23$ & $676.27 \pm 82.33$ & -2.63 & $0.02^{*}$ \\
ES & $529.53 \pm 97.99$ & $343.05 \pm 43.00$ & 2.41 & $0.03^{*}$ \\
VM & $2,485.44 \pm 807.46$ & $3,829.87 \pm 971.78$ & -5.65 & $0.00^{*}$ \\
VL & $1,535.53 \pm 466.78$ & $2,096.38 \pm 525.36$ & -3.33 & $0.00^{*}$ \\
\hline
\end{tabular}

Values are presented as number or mean \pm standard error.

SCM, sternocleidomastoid; SC, splenius capitis; $A B D$, rectus abdominis; EO, external abdominal oblique; TRA, transverse abdominis; ES, erector spinae; VM, vastus medialis; VL, vastus lateralis.

${ }^{*} P<0.05$, statistically significant difference.

\section{DISCUSSION}

We in this study used the EMG device to compare and analyze differences in muscular activities in the trunk and lower limbs during stair ascent based on ADIM. The results showed that the stair ascent when the ADIM was maintained increased the muscular activity of the transverse abdominal more than the ascent when the ADIM was not maintained, indicating a significant difference. Lee et al. (2013) reported that stair ascent accompanied with ADIM enhanced the core stability of the trunk when compared to the ascent without ADIM to significantly increase activities of the adnominal muscles that contributed to dynamic stabilization of the spine. Standaert et al. (2008) argued that internal abdominal pressure increased during functional activities and that the transverse abdominal as a stabilizer of the trunk was particularly activated by changes of postures. Unlike other abdominal muscles, the transverse abdominal is a deep muscle that continuously contracts (Cresswell et al., 1994), playing a crucial role in stabilization of the trunk (Richardson et al., 2004). The muscular activity of the transverse abdominal was higher instair ascent with ADIM maybe because ADIM induced contraction of the voluntary transverse abdominal muscle, measured and retrained its functions, and enhanced spinal stability (Stanton and Kawchuk, 2008).

Meanwhile, the muscular activity of the elector spinae was significantly low in stair ascent with ADIM, indicating a significant difference. Hides et al. (2006) reported that, when the transverse abdominal muscle pulled the abdominal walls, the muscle was thicker to enhance lumbar stability. According to Richardson et al. (2004), ADIM is a type of exercise that can functionally stimu- 
late the transverse abdominal, the internal oblique abdominal, and the multifidus muscles; the maneuver enhanced abdominal internal pressure to effectively perform lumbar stabilization by pulling the abdominal walls inward and contracting the abdominal muscles and induced simultaneous contraction of the muscles to reduce excessive lumbar lordosis or pelvic anterior tilt. For the erector spinae in this study, the activity of the lumbar extensors was likely to be reduced when the ADIM activated the abdominal muscles to enhance lumbar stability.

The vastus medialis and the vastus lateralis are the most important muscles around the knee joint. As the extensors of the joints, they provide stability of the lower limbs, especially of the knee joints, during standing or walking (Soderberg and Cook, 1983), the muscles effective in maintaining spinal stability by blocking internal external rotation. In this study, the two muscles increased in muscular activities with significant difference during stair ascent with ADIM. Konrad et al. (2001) reported that exercise for lumbar stability increased muscular activity of the legs, and other researchers argued that changes in leg and body postures during stair ascent affected muscular activity depending on the degrees of load applied (Choi et al., 2015; Feldwieser et al., 2012). Given that the activation of the abdominal muscles transmitted forces applied on the trunk to the legs (Neumann and Gill, 2002) and that the increased muscular activity of the legs induced an increase in the activity of the abdominal deep muscles, contributing to pelvic and spinal stability, by muscle synergy pattern, the results of the mentioned studies were consistent with those of this study (Choi et al., 2015).

In conclusion, according to the method with and without ADIM, the stair ascent was more active in the abdominal muscles contributing to stabilization of lumbar while maintaining the ADIM in the stair ascent, Which leg to an increase in the activity of the deep abdominal muscles, which contributed to the stabilization of the lumbar by the cooperative action of the muscles. Therefore, the stair ascent with ADIM maintained activated the abdominal muscles to control trunk balance and perform lumbar stability, simultaneously reducing excessive lumbar lordosis or pelvic anterior tilt which will help to improve the function of the musculoskeletal system.

\section{CONFLICT OF INTEREST}

No potential conflict of interest relevant to this article was reported.

\section{REFERENCES}

Akuthota V, Nadler SF. Core strengthening. Arch Phys Med Rehabil 2004; 85(3 Suppl 1):S86-92.

Bergmark A. Stability of the lumbar spine. A study in mechanical engineering. Acta Orthop Scand Suppl 1989;230:1-54.

Bjerkefors A, Ekblom MM, Josefsson K, Thorstensson A. Deep and superficial abdominal muscle activation during trunk stabilization exercises with and without instruction to hollow. Man Ther 2010;15:502-507.

Borschmann K, Pang MY, Bernhardt J, Iuliano-Burns S. Stepping towards prevention of bone loss after stroke: a systematic review of the skeletal effects of physical activity after stroke. Int J Stroke 2012;7:330-335.

Choi SA, Cynn HS, Yi CH, Kwon OY, Yoon TL, Choi WJ, Lee JH. Isometric hip abduction using a Thera-Band alters gluteus maximus muscle activity and the anterior pelvic tilt angle during bridging exercise. J Electromyogr Kinesiol 2015;25:310-315.

Cresswell AG, Oddsson L, Thorstensson A. The influence of sudden perturbations on trunk muscle activity and intra-abdominal pressure while standing. Exp Brain Res 1994;98:336-341.

Feldwieser FM, Sheeran L, Meana-Esteban A, Sparkes V. Electromyographic analysis of trunk-muscle activity during stable, unstable and unilateral bridging exercises in healthy individuals. Eur Spine J 2012; 21 Suppl 2:S171-186.

Galley PM, Foster AL. Human movement. Philadelphia (PA): Churchill Livingstone; 1987.

Hides J, Wilson S, Stanton W, McMahon S, Keto H, McMahon K, Bryant $\mathrm{M}$, Richardson C. An MRI investigation into the function of the transversus abdominis muscle during "drawing-in" of the abdominal wall. Spine (Phila Pa 1976) 2006;31:E175-178.

Hodges PW, Eriksson AE, Shirley D, Gandevia SC. Intra-abdominal pressure increases stiffness of the lumbar spine. J Biomech 2005;38:18731880.

Hodges PW, Gandevia SC. Activation of the human diaphragm during a repetitive postural task. J Physiol 2000;522 Pt 1:165-175.

Hodges PW, Richardson CA. Inefficient muscular stabilization of the lumbar spine associated with low back pain. A motor control evaluation of transversus abdominis. Spine (Phila Pa 1976) 1996;21:2640-2650.

Hubble RP, Naughton GA, Silburn PA, Cole MH. Trunk muscle exercises as a means of improving postural stability in people with Parkinson's disease: a protocol for a randomised controlled trial. BMJ Open 2014; 4:e006095.

Konrad P, Schmitz K, Denner A. Neuromuscular evaluation of trunktraining exercises. J Athl Train 2001;36:109-118.

Lee AY, Kim EH, Cho YW, Kwon SO, Son SM, Ahn SH. Effects of abdominal hollowing during stair climbing on the activations of local trunk 
stabilizing muscles: a cross-sectional study. Ann Rehabil Med 2013;37: 804-813.

Macedo LG, Maher CG, Latimer J, McAuley JH. Motor control exercise for persistent, nonspecific low back pain: a systematic review. Phys Ther 2009;89:9-25.

Marshall PW, Murphy BA. Core stability exercises on and off a Swiss ball. Arch Phys Med Rehabil 2005;86:242-249.

Meyer P, Kayser B, Mach F. Stair use for cardiovascular disease prevention. Eur J Cardiovasc Prev Rehabil 2009;16 Suppl 2:S17-18.

Neumann P, Gill V. Pelvic floor and abdominal muscle interaction: EMG activity and intra-abdominal pressure. Int Urogynecol J Pelvic Floor Dysfunct 2002;13:125-132.

Richardson CA, Hodges P, Hides J. Therapeutic exercise for lumbopelvic stabilization: a motor control approach for the treatment and prevention of low back pain. 2nd ed. Edinburgh (ED): Churchill Livingstone; 2004.

Richardson CA, Snijders CJ, Hides JA, Damen L, Pas MS, Storm J. The relation between the transversus abdominis muscles, sacroiliac joint mechanics, and low back pain. Spine (Phila Pa 1976) 2002;27:399-405.

Riener R, Rabuffetti M, Frigo C. Stair ascent and descent at different inclinations. Gait Posture 2002;15:32-44.

Saal JA. Dynamic muscular stabilization in the nonoperative treatment of lumbar pain syndromes. Orthop Rev 1990;19:691-700.

Soderberg GL, Cook TM. An electromyographic analysis of quadriceps femoris muscle setting and straight leg raising. Phys Ther 1983;63: 1434-1438.

Standaert CJ, Weinstein SM, Rumpeltes J. Evidence-informed management of chronic low back pain with lumbar stabilization exercises. Spine J 2008;8:114-120.

Stanton T, Kawchuk G. The effect of abdominal stabilization contractions on posteroanterior spinal stiffness. Spine (Phila Pa 1976) 2008;33:694701.

Yoon JY, An DH, Yoo WG, Kwon YR. Differences in activities of the lower extremity muscles with and without heel contact during stair ascent by young women wearing high-heeled shoes. J Orthop Sci 2009;14: 418-422. 